\title{
Use of a Simple Appliance to Prevent Mucosal Irritation by the Distractor Rod during Vertical Distraction: Case Report
}

\author{
Mehtap Muglalia \\ Nukhet Celebia \\ Samet Inala \\ Burak Bekcioglua \\ Burcu Bas ${ }^{a}$
}

\section{ABSTRACT}

Patient discomfort due to the excessive length of the distractor rod is one of the disadvantages of alveolar distraction osteogenesis. Although this seems to be a minor complication, it can cause severe mucosal irritation on the opposite arch, and uncontrolled forces can lead to the loosening of the screws and the inactivation of the distractor. The purpose of this case report is to introduce an acrylic appliance that is very useful in avoiding the mucosal irritation caused by the long distractor rod. The appliance is simple, effective, and easy to fabricate. (Eur J Dent 2008;2:204-207)

Key words: Vertical distraction appliance; Rod; Complication; Irritation.

\section{INTRODUCTION}

Vertical alveolar distraction osteogenesis (ADO) has received considerable interest in terms of an extremely resorbed edentulous mandible as a way to augment bone prior to implant placement. Compared with the conventional techniques of bone grafting and guided bone regeneration, ADO offers the advantages of decreased bone resorption, a lower rate of infection, and no donor

a Department of Oral and Maxillofacial Surgery, Faculty of Dentistry, Ondokuz Mayis University, Samsun, TURKEY

- Corresponding author: Dt. Burak Bekcioglu Department of OMS, Faculty of Dentistry, Ondokuz Mayis University, 55139, Kurupelit, Samsun, Turkey. Phone: +90 $3623121919-3480$

Fax: +90 3624576032

E-mail: burakbekciogluahotmail.com site morbidity; $;^{1,2}$ also, tissue is gained..$^{1,3,4}$

Disadvantages consist of the difficulty in controlling the segments, a lack of patient cooperation and the need for more office visits, and the cost of the device. ${ }^{5-8}$ Common complications related to distraction osteogenesis are basal bone or transport segment fracture, fixation screw loss, nonunion, premature consolidation, wound dehiscences, lingual positioning of the transport segment, resorption of the transport segment, excessive length of the threaded rod, neurological alterations, and distractor fractures. ${ }^{79-11}$

In addition to these complications, the irritation of the oral mucosa on the opposite jaw caused by the distractor rod can be mentioned. The purpose of this study is to introduce a simple appliance to prevent distractor fracture and the irritation caused by the distractor rod. 


\section{CASE REPORT}

A 60-year-old woman, who was completely edentulous in both the maxilla and mandible, was referred to our clinic with a complaint of poor retention of her conventional lower denture. Clinical and radiographic examinations revealed severe atrophy in the mandible. Resorption in the mandible did not allow insertion of the implants to the optimal length. To achieve sufficient bone height for the implants, vertical alveolar distraction osteogenesis was planned in the anterior region of the mandible. She had no compromising medical factors that would affect the surgical procedure.

The surgery was performed under local anesthesia. The mandibular ridge between the mental foramina was exposed by a crestal incision and the raising of a full-thickness mucoperiosteal flap. The mental nerves were carefully located and preserved. The interforaminal osteotomy was delineated. The distraction device (Q-Multitractor, Trinon ${ }^{\circ}$, Germanyl was mounted and removed, and the osteotomy completed. Finally, the distraction device was replaced in its previous position; segment mobilization was controlled by activating the distractor intraoperatively. The mucoperiosteal flap was then closed primarily. Antibiotic, analgesic, and mouthwash were prescribed.

After one week of latency, distraction was started at a rate of $0.3 \mathrm{~mm}$ twice a day. On the fourth day of distraction, the patient complained of a pain in her maxillary anterior region, especially in the mornings. She also suffered from irritation caused by the distraction rod on the same area during oral functions. Clinical

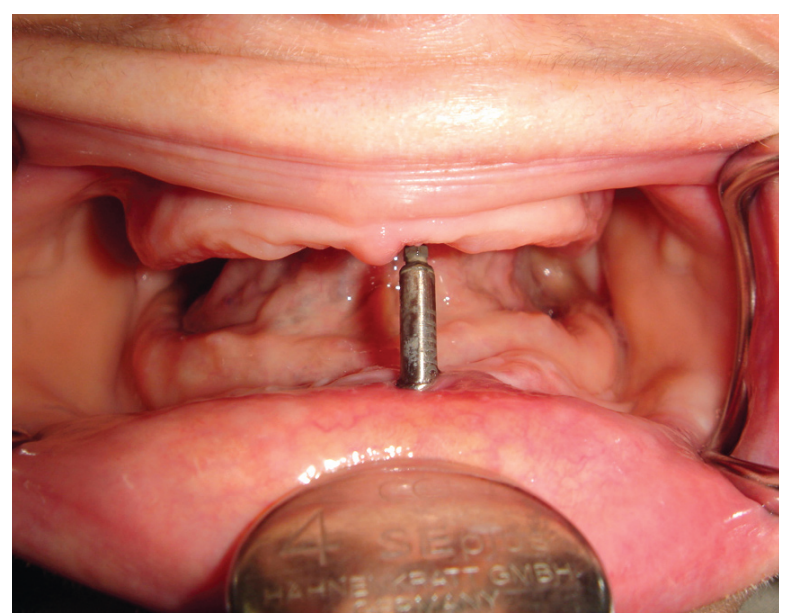

Figure 1. Distractor rod in contact with the upper crest. examination showed that the rod of the distractor was in contact with the upper crest (Figure 1). We thought that the irritation and pain could be the result of parafunctional oral activities during sleep. Therefore, we designed a simple appliance (Figure 2) to eliminate the patient's discomfort due to the distractor rod and the risk of distractor fracture. The appliance had extensions on the posterior region that were in contact with the posterior mandibular alveolar ridge. Soft relining material (Mollosil, Detax $®$, Germany) was applied to the extensions. To prevent the exposure of unattempted forces on the distracted alveolar segment, the extensions were designed away from the anterior region. It was suggested to the patient that she should use the appliance only at night for three months (Figure 3). During this period, the patient's complaints ceased once she started to use the appliance; no TMJ or salivary problem was observed. After the distraction period, implants were inserted (Figures 4 and 5) and prosthetic rehabilitation was completed six months later.

\section{DISCUSSION}

Patients with a severely resorbed edentulous mandible often suffer from problems with the lower denture. Dental implants have been shown to provide a reliable basis for fixed and removable prostheses; however, unfavorable local conditions of the alveolar ridge may provide insufficient bone volume for the placement of implants. Reconstructive pre-prosthetic surgery has changed from surgery aimed to provide a sufficient osseous and mucosal support for a conventional denture into surgeryaimed to provide

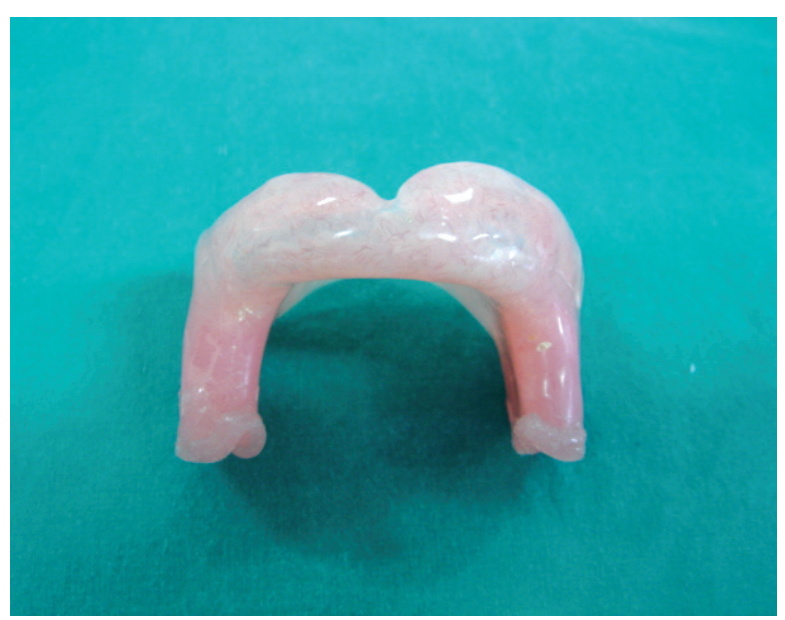

Figure 2. The view of acrylic appliance. 
a sufficient bone volume to enable implants to be placed at the most optimal positions from a prosthetic point of view. ${ }^{12}$ Various techniques and materials have been developed to increase mandibular height before implant placement, including augmentation with autogen bone grafts or bone substitutes and combinations of both. Major drawback of these procedures is the donor area morbidity and nerve disturbances. ${ }^{13}$ The placement of short endosseous implants is another option to treat the extremely resorbed mandible, however, the failure rate of short implants is higher than that of longer implants and complications are more common. ${ }^{14}$

Alveolar distraction osteogenesis offers advantages over other crest reconstruction techniques. ${ }^{5,7}$ The technique results in the rapid natural formation of vascularise bone between the basal mandibular portion and transport fragment which eliminates the need to harvest bone, thus reducing morbidity, ${ }^{15}$ but some complications can occur during the activation phase or postoperative period. ${ }^{5,7}$ Possible complication of distraction

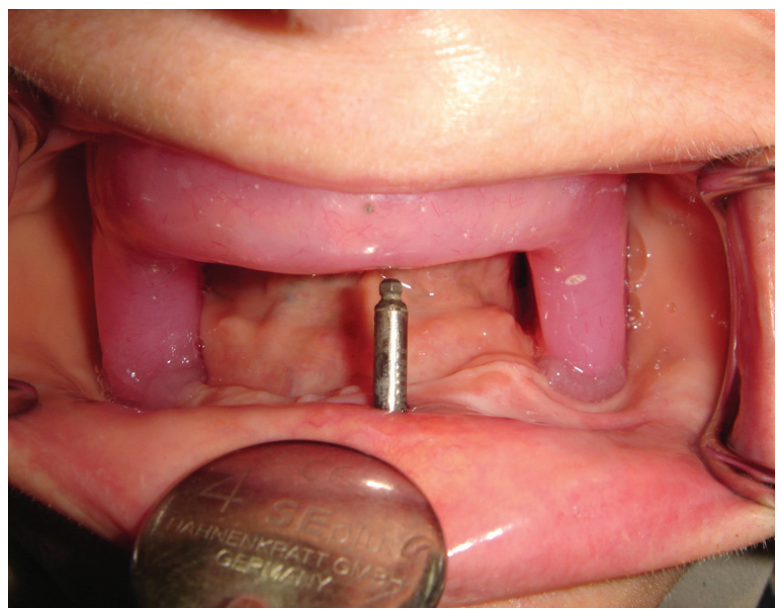

Figure 3. Intraoral view of the appliance.

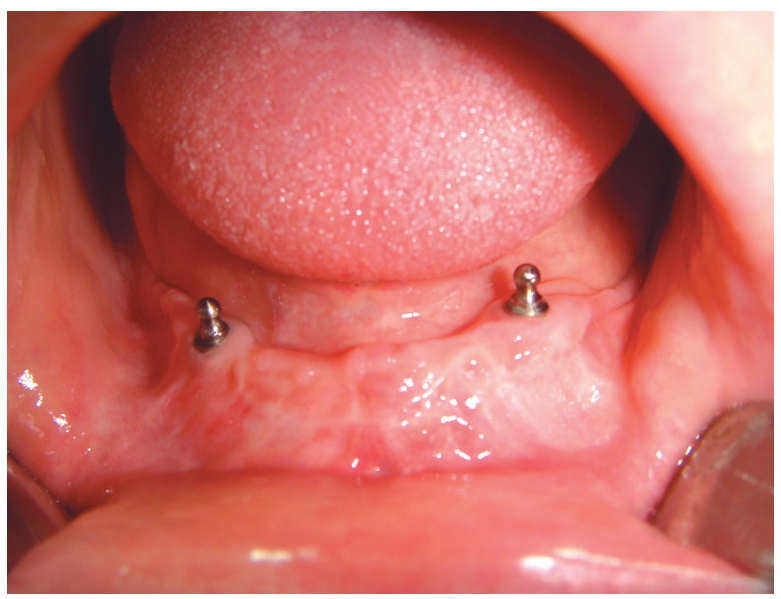

Figure 4. Intraoral view of the implants. techniques for the edentulous (severely resorbed) mandible are fracture of the mandible, infection, and necrosis of the superior fragment, but such complications are rarely reported in the literature. ${ }^{12}$ In this case, no intraoperative complications were observed, while a different postoperative complication, caused by the rod of the distraction device, was encountered.

The patient complained about discomfort due to the distraction device because of its long rod, which irritated her anterior maxillary region. The excessive length of the threaded rod is a significant problem, because the distractor must remain in the patient's mouth for at least 14 weeks. ${ }^{16}$ She complained about severe pain in her anterior maxilla, especially in the mornings, probably because of uncontrolled oral functions while sleeping. To manage this problem, we designed a simple prosthesis-like appliance to eliminate the interference between the rod and the oral mucosa. By keeping the edges of the appliance away from the osteotomy lines, we prevented the application of undesired forces to the mobile bone segment.

Generally, the rods of distraction devices are longer than patient's vertical rest position, so the irritation of mucosa due to the rod is an expected complication. In this case, irritation of the oral mucosa of the opposite arch is due to the distractor type we chose. It must be controlled, whether or not the rod is in contact with the opposite jaw, while the distractor is mounted. But, in some cases, especially in Class II patients, due to anatomic variations, it is not possible to avoid this situation by bending the distractor plaques. Although this can be considered a minor

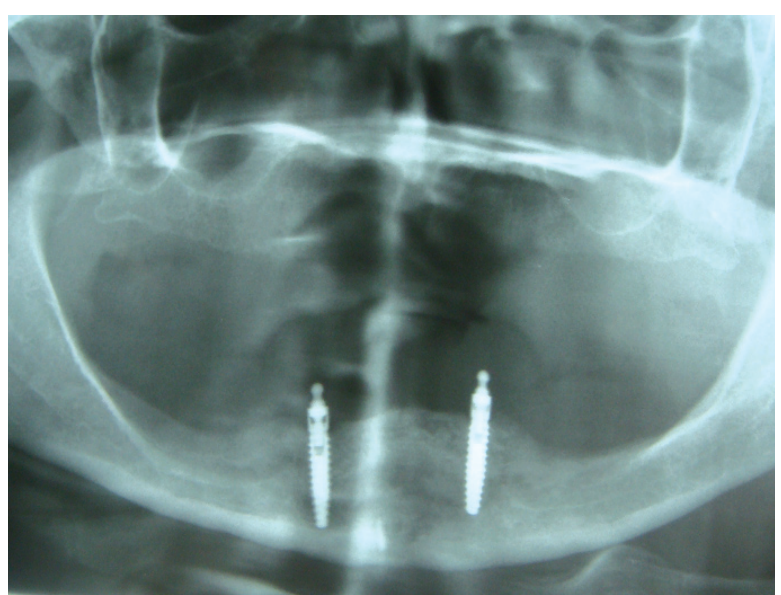

Figure 5. Panoramic view after insertion of the implants. 
complication, it can lead to the loosening of screws and even the inactivation of the distractor because of the excessive force that comes to the rod of the distractor. With endo distraction devices like Mondeal $\AA$ and Krenkel $®$, this problem can be avoided and in some distraction devices, this problem is readily solved because the threaded rod can be cut without affecting its function. ${ }^{16}$ However, in our distractor, we did not have this choice.

The appliance was made of acrylic and covered with soft relining material to prevent any irritation to oral mucosa. However, in some patients who have atrophied mandibles which the nerve is situated on top of the mandible, use of the appliance can be contraindicated due to the high pressure of the extensions to posterior mandible. Other possible complications of the appliance are salivary problems like hypersalivation or xerostomia, as in the devices used in cases of snore and sleep apnea, but we did not encounter such problems in our case.

In this study, we developed a simple and comfortable appliance to manage these complications. Although we did not experience any TMJ problems, the possibility of this complication should also be kept in mind and TMJ examinations performed in control visits.

\section{REFERENCES}

1. Horiuchi K, Uchida H, Yamamoto K, Hatano N. Anteroinferior distraction of the atrophic subtotalmaxillary alveolus for implant placement: A case report. Int J Oral Maxillofac Implants 2002;17:416-423.

2. Urbani G. Alveolar distraction before implantation: A report of five cases and a review of the literature. Int J Periodontics Restorative Dent 2001;21:569-579.

3. Jensen OT, Cockrell R, Kuhlke L, Reed C. Anterior maxillary alveolar distraction osteogenesis: A prospective 5- year clinical study. Int J Oral Maxillofac Implants 2002;17:52-68.

4. Raghoebar GM, Heydenrijk K, Vissink A. Vertical distraction of the severely resorbed mandible. The Groningen distraction device. Int J Oral Maxillofac Surg 2000;29:416420.

5. Garcia-Garcia A, Martin-Somoza M, Gandara-Vila P, Maceiras JL. Minor complications arising in alveolar distraction osteogenesis. JOral Maxillofac Surg 2002;60:496501.
6. Hidding J, Lazar F, Zöller JE. Initial outcome of vertical distraction osteogenesis of the atrophic alveolar ridge. Mund Kiefer Gesichtschir 1999;3:S79-83.

7. Uckan S, Haydar SG, Dolanmaz D. Alveolar distraction: analysis of 10 cases. Oral Surg Oral Med Oral Pathol Oral Radiol Endod 2002;94:561-565.

8. Van Strijen PJ, Breuning KH, Becking AG, Perdijk FB, Tuinzing DB. Complications in bilateral mandibular distraction osteogenesis using internal devices. Oral Surg Oral Med Oral Pathol Oral Radiolo Endod 2003;96:392-397.

9. McAllister BS. Histologic and radiographic evidence of vertical ridge augmentation utilizing distraction osteogenesis: 10 consecutively placed distractors. $J$ Periodontol 2001;72:1767-1779.

10. Mazzonetto R, Torezan JF. Potential complications during alveolar distraction osteogenesis. J Oral Maxillofac Surg 2003;61:85

11. McAllister BS, Gaffaney TE. Distraction osteogenesis for vertical bone augmentation prior to oral implant reconstruction. Periodontol 2000 2003;33:54-66.

12. Stellingsma C, Vissink A, Meijer HJ, Kuiper C, Raghoebar GM. Implantology and the severely resorbed edentulous mandible. Crit Rev Oral Biol Med 2004;15:240-248.

13. de Koomen HA, Stoelinga PJ, Tideman H, Huybers TJ. Interposed bone-graft augmentation of the atrophic mandible (a progress report). J Maxillofac Surg 1979;7:129135.

14. Stellingsma C, Raghoebar GM, Meijer HJ, Batenburg RH. Reconstruction of the extremely resorbed mandible with interposed bone grafts and placement of endosseous implants. A preliminary report on outcome of treatment and patients' satisfaction. Br J Oral Maxillofac Surg 1998;36:290295.

15. Bianchi A, Felice P, Lizio G, Marchetti C. Alveolar distraction osteogenesis versus inlay bone grafting in posterior mandibular atrophy: a prospective study. Oral Surg Oral Med Oral Pathol Oral Radiolo Endod 2008;105:282-292.

16. Garcia AG, Martin MS, Vila PG, Maceiras JL. Minor complications arising in alveolar distraction osteogenesis. J Oral Maxillofac Surg 2002;60:496-501. 